\title{
THE KOLMOGOROV EQUATION IN THE STOCHASTIC FRAGMENTATION THEORY AND BRANCHING PROCESSES WITH INFINITE COLLECTION OF PARTICLE TYPES
}

\author{
R. YE. BRODSKII AND YU. P. VIRCHENKO \\ Received 26 June 2005; Accepted 1 July 2005
}

The stochastic model for the description of the so-called fragmentation process in frameworks of Kolmogorov approach is proposed. This model is represented as the branching process with continuum set $(0, \infty)$ of particle types. Each type $r \in(0, \infty)$ corresponds to the set of fragments having the size $r$. It is proved that the branching condition of this process represents the basic equation of the Kolmogorov theory.

Copyright (c) 2006 R. Ye. Brodskii and Yu. P. Virchenko. This is an open access article distributed under the Creative Commons Attribution License, which permits unrestricted use, distribution, and reproduction in any medium, provided the original work is properly cited.

\section{Introduction}

There are various natural processes that represent the evolution during time of solid state media in the form of some successive subdivisions of all its connected parts to smaller parts having random forms and volumes, and, consequently, masses and/or chemical compositions. In statistical physics they are called the fragmentation processes. It is clear that such processes may have an adequate mathematical description only on the basis of some concepts of probability theory. Notice also that even the description of each separate random state of such a physical system, that is, the construction of the space $\Omega$ of elementary events, meets with some fatal troubles. Moreover, it is not clear what principles are necessary to use in order to construct adequate stochastic dynamics in the form of a random process in the space $\Omega$. On the other hand, it seems unreasonable to think that the models of the great variety of physical fragmentation processes may be done on the basis of some relatively simple probabilistic scheme.

In the initial work of Kolmogorov on statistical fragmentation theory (Kolmogorov [2]), an approach to probabilistic description of fragmentation processes is proposed. It is based on the use of states characterizing the dynamical subdivision system at each specified time instant $t$ by a random function $\tilde{N}(r, t)$ that takes values only in $\mathbb{N}_{+}$and depends only on the unique nonnegative parameter $r$, which we will further call the 
fragment size. Each value of this function represents the number of fragments at time instant $t$ with sizes being not greater than $r$. Therefore, in the framework of this approach, the mathematical model of fragmentation is represented by a random process $\left\{\tilde{N}(r, t) ; t \in \mathbb{R}_{+}=(0, \infty)\right\}, r \in \mathbb{R}_{+}$with values in $\mathbb{R}_{+}^{\mathbb{N}_{+}}$. In Kolmogorov [2] a simple evolution equation for mathematical expectations $\mathbf{E} \tilde{N}(r, t)$ (we consider the discrete time case) is derived. It has Markovian type and is constructed in terms of mathematical expectation $\mathbf{E} \tilde{\mathcal{\nu}}\left(r \mid r^{\prime} ; t\right)$ of the other random function $\tilde{\nu}\left(r \mid r^{\prime} ; t\right): \mathbb{R}_{+} \times \mathbb{R}_{+} \times \mathbb{N}_{+} \mapsto \mathbb{N}_{+}$that is the random number of fragments with sizes not greater than $r$ and generated at time instant $t$ from a specified randomly chosen fragment having the size $r^{\prime}$. This equation has the following form:

$$
\mathbf{E} \tilde{N}(r, t+1)=\int_{0}^{1} \mathbf{E} \tilde{N}\left(\frac{r}{k}, t\right) d S(k, t)
$$

under the assumption that the function $\mathbf{E} \tilde{\mathcal{V}}\left(r \mid r^{\prime} ; t\right) \equiv S(k, t)$ depends only on the fraction $k=r / r^{\prime}$. Thus, the model formulated in Kolmogorov [2] is obtained on the basis of some phenomenological reasons as it is said in physical literature. These reasons are based on the concept of "the average field" that is often in use in statistical physics. Further, in Kolmogorov [2], it is proved that the integral limit theorem for the distribution function

$\mathbf{E} \tilde{N}(r, t) / \mathbf{E} \tilde{N}(\infty, t)$ takes place under the assumption that the function $\mathbf{E} \tilde{\mathcal{v}}(k, t)$ does not depend on time and that its second "logarithmi" moment in the variable $k$ is finite. It may be considered as the marginal one-dimensional probabilistic distribution of the random process $\{\tilde{r}(t) ; t \in[0, \infty)\}$, with nonnegative trajectories $\tilde{r}(t)$ and, physically, as the size of randomly chosen fragment from the whole system at time $t$.

Here we will not discuss the physical question of applicability of the abovementioned approach of the mathematical modelling to some real physical fragmentation processes. Our problem consists of the ground of (1.1) on the basis of an explicit construction of the random process $\{\tilde{N}(r, t) ; t \in[0, \infty)\}$. The idea of such a ground has been stated in the cited work. But it seems that the consequent authors (see, e.g., the fundamental work (Filippov [1])) have not taken into account the great importance of this idea to realize it. From our point of view such an explicit construction of the mathematical model of a higher level, in the frameworks of which the main master (1.1) of Kolmogorov theory can be proved as a mathematical statement, may represent the important base for constructing more complicated (and, therefore, more adequate) models in the fragmentation theory.

\section{Mathematical model description}

Specify a number $\Delta>0$. Further, divide the positive part $[0, \infty)$ of the real line into the sequence of disjoint half-open intervals $\left\langle[i \Delta,(i+1) \Delta) ; i \in \mathbb{N}_{+}\right\rangle$being open from the right. Their union coincides with $[0, \infty)$. Introduce the random process $\mathfrak{N}_{\Delta}$ with discrete time and with values in the set $\mathbb{N}_{+}^{\mathbb{N}_{+}}$. The sampling space of this process consists of some random collections of functions $\left\{\left\langle\tilde{\nu}_{i}(t) ; i \in \mathbb{N}_{+}\right\rangle ; t \in \mathbb{N}_{+}\right\}$. Each function takes its values in $\mathbb{N}_{+}$. By its sense, each function $\widetilde{v}_{i}(t), i=0,1,2, \ldots$, represents the number of fragments, 
having random sizes, that belong to half-interval $[i \Delta,(i+1) \Delta)$. Define the process $\mathfrak{N}_{\Delta}$ as the Markov branching one with discrete time (see Sevast'ianov [3]) (the Markov chain). Generally speaking, it is inhomogeneous in time. Besides, it has an infinite collection $\mathbb{N}_{+}$ of particle types. The last words are taken from the terminology of branching random process theory. In our problem fragments with specified size $r$ are the particles of some definite type from the point of view of this terminology.

Since the countable set $\mathbb{N}_{+}^{\mathbb{N}_{+}}$is the process state space, then for each time instant $t \in \mathbb{N}_{+}$ the conditional probabilities

$$
Q\left(m_{i}, i \in \mathbb{N}_{+} \mid n_{j}, j \in \mathbb{N}_{+} ; t\right)=\operatorname{Pr}\left\{\tilde{v}_{j}(t+1)=n_{j}, j \in \mathbb{N}_{+} \mid \tilde{v}_{i}(t)=m_{i}, i \in \mathbb{N}_{+}\right\}
$$

of transitions form an infinite matrix when arguments $n_{j}, m_{i} \in \mathbb{N}_{+}, i, j \in \mathbb{N}_{+}$are changed. The matrix (2.1) of transition conditional probabilities defines completely the Markov chain with countable set of states. In particular, it defines the evolution of onedimensional marginal probability distribution of this chain

$$
P\left(n_{i}, i \in \mathbb{N}_{+} ; t\right)=\operatorname{Pr}\left\{\widetilde{v}_{i}(t)=n_{i}, i \in \mathbb{N}_{+}\right\}
$$

namely, it is defined uniquely by the Markov chain equation

$$
P\left(n_{j}, j \in \mathbb{N}_{+} ; t+1\right)=\sum_{\left\{m_{i}\right\}} P\left(m_{i}, i \in \mathbb{N}_{+} ; t\right) Q\left(m_{i}, i \in \mathbb{N}_{+} \mid n_{j}, j \in \mathbb{N}_{+} ; t\right),
$$

where, here and below, the symbol of summation means that it is done with respect to all possible distributions of "filling numbers," that is, with respect to all collections $\left\langle m_{i}, i \in \mathbb{N}_{+}\right\rangle \in \mathbb{N}_{+}^{\mathbb{N}_{+}}$. For the matrix $Q\left(m_{i}, i \in \mathbb{N}_{+} \mid n_{j}, j \in \mathbb{N}_{+} ; t\right), n_{j}, m_{i} \in \mathbb{N}_{+} i, j \in \mathbb{N}_{+}$, we will use also the shorter notation $Q\left(m_{i} \mid n_{j} ; t\right)$. It is constructed for the Markov branching process by the following way. Define the function $q_{l}\left(k_{j}, j \in \mathbb{N}_{+} ; t\right) \equiv q_{l}\left(k_{j} ; t\right)$. It represents the probability of the event that describes the fact that a specified fragment with size $l$ (i.e., its size $r$ belongs to the half-interval $[l \Delta,(l+1) \Delta))$ gets at the time instant $t$ to the set of fragments and this set is characterized by the collection of filling numbers $\left\langle k_{j} ; j \in \mathbb{N}_{+}\right\rangle$. In this case, of course, this probability is not zero only if $k_{j}=0$ at $j>l$. Thus, $q_{l}\left(k_{j}, j \in \mathbb{N}_{+} ; t\right)$ is the probability of the fact that the random function $\widetilde{\nu}_{l, j}(t): \mathbb{N}_{+} \times \mathbb{N}_{+} \times \mathbb{N}_{+} \mapsto \mathbb{N}_{+}$takes value $k_{j}$. The function is the number of fragments with sizes $j$ that are formed from the specified fragment with size $l$ at the time instant $t$; here, the second argument $j$ is not greater than $l$. Further, we introduce the random function $\tilde{\eta}: \mathbb{N}_{+} \times \mathbb{N}_{+} \times \mathbb{N} \times \mathbb{N}_{+} \mapsto$ $\mathbb{N}_{+}, \tilde{\eta}_{l, j}(m ; t)=\widetilde{v}_{l, j}^{(1)}(t)+\widetilde{v}_{l, j}^{(2)}(t)+\cdots+\widetilde{v}_{l, j}^{(m)}(t)$ for each pair $l, j \in \mathbb{N}_{+}$. It is the sum of $m \in \mathbb{N}$ statistically independent random functions $\widetilde{v}_{l, j}^{(1)}(t), \widetilde{\nu}_{l, j}^{(2)}(t), \ldots, \widetilde{v}_{l, j}^{(m)}(t)$ and it represents the set of filling numbers on sizes $j$ of fragments formed by subdivision from $m$ identical fragments having the size $l$ at the time instant $t$. In such a definition of the branching condition that describes the disintegration of fragments having the size $l$, the 
4 The Kolmogorov equation in the stochastic fragmentation

individuality of each fragment is lost, that is, for each fixed fragment in the final state we do not take into account the fact, from which fragment of the size $l$ appeared as a result of the disintegration. Due to the given definition of the random function $\tilde{\eta}_{l}\left(m, k_{j}, j \in \mathbb{N}_{+} ; t\right)$, its probability distribution $q_{l}\left(m \mid k_{j}, j \in \mathbb{N}_{+} ; t\right)$ is defined by the $m$-multiple convolution of the probability distribution collection $q_{l}\left(k_{j}^{(i)}, j \in \mathbb{N}_{+} ; t\right), i=1, \ldots, m$,

$$
q_{l}\left(m \mid k_{j}, j \in \mathbb{N}_{+} ; t\right)=\sum_{\substack{k_{j}^{(i)} \geq 0, i=1, \ldots, m, k_{j}^{(1)}+\cdots+k_{j}^{(m)}=k_{j}, j \in \mathbb{N}_{+}}} \prod_{i=1}^{m} q_{l}\left(k_{j}^{(i)}, j \in \mathbb{N}_{+} ; t\right) .
$$

Indeed, the probability $q_{l}\left(m \mid k_{j}, j \in \mathbb{N}_{+} ; t\right)$ is equal to zero if there exists $j \in \mathbb{N}_{+}, j>l$ such that the inequality $k_{j} \neq 0$ is valid.

At last, the matrix $Q\left(m_{i} \mid n_{j} ; t\right)$ is determined by the formula

$$
\begin{aligned}
& Q\left(m_{i}, i \in \mathbb{N}_{+} \mid n_{j}, j \in \mathbb{N}_{+} ; t\right) \\
& =\sum_{k_{i j} \geq 0 ; i, j \in \mathbb{N}_{+}}\left[\prod_{i=0}^{\infty} q_{i}\left(m_{i} \mid k_{i l}, l \in \mathbb{N}_{+} ; t\right)\right]\left[\prod_{j=0}^{\infty} \delta\left(n_{j}-\sum_{l: l \geq j} k_{l j}\right)\right],
\end{aligned}
$$

where $\delta\left(n-n^{\prime}\right) \equiv \delta_{n, n^{\prime}}$ is the Kronecker symbol and the summation is done on all twoplaced functions $k_{i j}: \mathbb{N}_{+} \times \mathbb{N}_{+} \mapsto \mathbb{N}_{+}$. The sense of the integer matrix is that it determines the fragment numbers with the size $j$ that are formed from all fragments with size $i$.

The matrix $Q\left(m_{i} \mid n_{j} ; t\right)$ and the probability distribution $P\left(n_{j}, j \in \mathbb{N}_{+} ; 0\right)$ determine the random process $\mathfrak{N}_{\Delta}$ completely as well as (in particular) its characteristic functional $\Psi_{\Delta}[u]: \mathbb{S}_{\infty}^{\mathbb{N}_{+}}\left(\mathbb{R}_{+}\right) \mapsto \mathbb{C}$, the value of which is determined as

$$
\Psi_{\Delta}[u]=\operatorname{E} \exp \left(i \sum_{t=0}^{\infty} \sum_{j=0}^{\infty} \tilde{\nu}_{j}(t) \int_{j \Delta}^{(j+1) \Delta} u_{t}(x) d x\right)
$$

for each function sequence $u_{t}(x), t=0,1,2, \ldots$ from the space $\mathbb{S}_{\infty}\left(\mathbb{R}_{+}\right)$of compactly supported functions being infinitely differentiable on $\mathbb{R}_{+}$. Values of the functional exist due to the support compactness in $x$ of the functions $u_{t}(x)$.

Now we give the definition of the random process $\mathfrak{N}$ with values in $\mathbb{R}_{+}^{\mathbb{N}_{+}}$that describes the fragmentation. We will define it as the generalized random process generated by the process sequence $\mathfrak{N}_{\Delta}$ at $\Delta \rightarrow 0$. 
Definition 2.1. Generalized random process $\mathfrak{N}$ with the characteristic functional $\Psi[u]$, determined by the limit

$$
\Psi[u]=\lim _{\Delta \rightarrow 0} \Psi_{\Delta}[u]
$$

for each function $u_{t}(x) \in \mathbb{S}_{\infty}^{\mathbb{N}_{+}}\left(\mathbb{R}_{+}\right)$, is called the random Kolmogorov fragmentation process.

\section{Equation for the generating function}

Introduce the space $\mathbb{S}_{\infty}\left(\mathbb{N}_{+}\right)$of infinite bounded sequences where each of them has zero components beginning from a number. Further, we will imply that such sequences $X$ have only nonnegative components. The set of all those sequences forms the cone in $\mathbb{S}_{\infty}\left(\mathbb{N}_{+}\right)$.

We also introduce the sequence $\mathrm{G}[X, t]=\left\langle g_{l}[X, t] ; l \in \mathbb{N}_{+}\right\rangle$whose components are generating functions of probability distributions $q_{l}\left(k_{j} ; t\right), l \in \mathbb{N}_{+}$,

$$
g_{l}[X, t]=\sum_{\left\{k_{j}\right\}}\left(\prod_{j=0}^{\infty} x_{j}^{k_{j}}\right) q_{l}\left(k_{j}, j \in \mathbb{N}_{+} ; t\right) .
$$

Formally, they are functions of countable set of variables. However, due to the variable $\left\langle k_{j} ; j \in \mathbb{N}_{+}\right\rangle$in the probability distribution is a finite sequence, really, the function $g_{l}[X, t]$ depends only on finite components in $X$. Each $l$ th function depends on $l$ variables where $l$ is determined by the maximal number $j$ among nonzero components in $k_{j} \neq 0$.

Now compute the sums

$$
\begin{aligned}
h_{l}^{(n)}[X, t] & =\sum_{\left\{k_{j}\right\}}\left(\prod_{j=0}^{\infty} x_{j}^{k_{j}}\right) q_{l}\left(n \mid k_{j}, j \in \mathbb{N}_{+} ; t\right) \\
& =\sum_{\left\{k_{j}\right\}}\left(\prod_{j=0}^{\infty} x_{j}^{k_{j}}\right) \sum_{k_{j}^{(i)} \geq 0, i=1, \ldots, n,} \prod_{i=1}^{n} q_{l}\left(k_{j}^{(i)}, j \in \mathbb{N}_{+} ; t\right) \\
& =\sum_{\left\{k_{j}\right\}}^{(1)}+\cdots+k_{j}^{(n)}=k_{j}, j \in \mathbb{N}_{+} \\
& =\sum_{k_{j}^{(i)} \geq 0, i=1, \ldots, n,} \prod_{i=1}^{n}\left(\prod_{j=0}^{\infty} x_{j}^{k_{j}^{(i)}}\right) q_{l}\left(k_{j^{\prime}}^{(i)}, j^{\prime} \in \mathbb{N}_{+} ; t\right) \\
& =\prod_{i=1}^{n}\left[\sum _ { \{ k _ { j } ^ { ( i ) } \} } \left(\prod_{j=0}^{\infty} x_{j}^{k_{j}^{(i)}}=k_{j}, j \in \mathbb{N}_{+}\right.\right. \\
& \left.\left.\prod_{i=1, \ldots, n, j \in \mathbb{N}_{+}}^{n}\left(\prod_{j=0}^{\infty} x_{j}^{k_{j}^{(i)}}\right) q_{l}\left(k_{j^{\prime}}^{(i)}, j^{\prime} \in \mathbb{N}_{+}^{(i)} ; t\right)\right]=\prod^{\prime} \in \mathbb{N}_{+} ; t\right)
\end{aligned}
$$


6 The Kolmogorov equation in the stochastic fragmentation

Finally, compute the sum

$$
\begin{aligned}
h\left[m_{i},\right. & \left.i \in \mathbb{N}_{+} \mid n_{j}, j \in \mathbb{N}_{+} ; X, t\right] \\
& =\sum_{\left\{n_{j}\right\}}\left(\prod_{j=0}^{\infty} x_{j}^{n_{j}}\right) Q\left(m_{i}, i \in \mathbb{N}_{+} \mid n_{j^{\prime}}, j^{\prime} \in \mathbb{N}_{+} ; t\right) \\
& =\sum_{\left\{n_{j}\right\}} \sum_{k_{i j} \geq 0 ; i, j \in \mathbb{N}_{+}}\left[\prod_{j=0}^{\infty} x_{j}^{n_{j}} \delta\left(n_{j}-\sum_{l: l \geq j} k_{l j}\right)\right]\left[\prod_{i=0}^{\infty} q_{i}\left(m_{i} \mid k_{i l}, l \in \mathbb{N}_{+} ; t\right)\right] \\
& =\sum_{k_{i j} \geq 0 ; i, j \in \mathbb{N}_{+}}\left[\prod_{i=0}^{\infty}\left(\prod_{j=0}^{\infty} x_{j}^{k_{i j}}\right) q_{i}\left(m_{i} \mid k_{i l}, l \in \mathbb{N}_{+} ; t\right)\right] \\
& =\prod_{i=0}^{\infty}\left[\sum_{k_{i j} \geq 0 ; j \in \mathbb{N}_{+}}\left(\prod_{j=0}^{\infty} x_{j}^{k_{i j}}\right) q_{i}\left(m_{i} \mid k_{i l}, l \in \mathbb{N}_{+} ; t\right)\right] \\
& =\prod_{i=0}^{\infty} h_{i}^{\left(m_{i}\right)}[X, t]=\prod_{i=0}^{\infty} g_{i}^{m_{i}}[X, t],
\end{aligned}
$$

where we use the rule

$$
\prod_{j=0}^{\infty} x_{j}^{n_{j}}=\prod_{j=0}^{\infty} \prod_{i: i \geq j} x_{j}^{k_{i j}}=\prod_{i=0}^{\infty} \prod_{j: i \geq j} x_{j}^{k_{i j}},
$$

and also we take into account that probabilities $q_{i}\left(m_{i} \mid k_{i l}, l \in \mathbb{N}_{+} ; t\right)$ are not zero only if $k_{i j}=0$ at $i<j$.

After these preparatory computations, introduce the generating function $\mathbf{H}_{t}[X]$ of the one-dimensional probability distribution $P\left(n_{j}, j \in \mathbb{N}_{+} ; t\right)$ of the Markov chain at the time $t$ according to the formula

$$
\mathbf{H}_{t}[X]=\sum_{\left\{n_{j}\right\}}\left(\prod_{j=0}^{\infty} x_{j}^{n_{j}}\right) P\left(n_{j}, j \in \mathbb{N}_{+} ; t\right) .
$$

Then, applying the operation $\sum_{\left\{n_{j}\right\}}\left(\prod_{j=0}^{\infty} x_{j}^{n_{j}}\right)$ to equation of motion (2.3) and using (3.3), we find the motion equation of the generating function $\mathbf{H}_{t}[X]$,

$$
\begin{aligned}
\mathbf{H}_{t+1}[X] & =\sum_{\left\{n_{j}\right\}}\left(\prod_{j=0}^{\infty} x_{j}^{n_{j}}\right) \sum_{\left\{m_{i}\right\}} P\left(m_{i}, i \in \mathbb{N}_{+} ; t\right) Q\left(m_{i}, i \in \mathbb{N}_{+} \mid n_{j}, j \in \mathbb{N}_{+} ; t\right) \\
& =\sum_{\left\{m_{i}\right\}} P\left(m_{i}, i \in \mathbb{N}_{+} ; t\right) \sum_{\left\{n_{j}\right\}}\left(\prod_{j=0}^{\infty} x_{j}^{n_{j}}\right) Q\left(m_{i}, i \in \mathbb{N}_{+} \mid n_{j}, j \in \mathbb{N}_{+} ; t\right) \\
& =\sum_{\left\{m_{i}\right\}} P\left(m_{i}, i \in \mathbb{N}_{+} ; t\right) h\left[m_{i}, i \in \mathbb{N}_{+} \mid n_{j}, j \in \mathbb{N}_{+} ; X, t\right] \\
& =\sum_{\left\{m_{i}\right\}} P\left(m_{i}, i \in \mathbb{N}_{+} ; t\right)\left(\prod_{i=0}^{\infty} g_{i}^{m_{i}}[X, t]\right)=\mathbf{H}_{t}[\mathbf{G}[X, t]],
\end{aligned}
$$

where $\mathbf{G}[X, t]=\left\langle g_{l}[X, t] ; l \in \mathbb{N}_{+}\right\rangle$. 
Thus, we have proved the following theorem.

Theorem 3.1. Generating function $\mathbf{H}_{t}[X]$ of the probability distribution $P\left(n_{j}, j \in \mathbb{N}_{+} ; t\right)$ is governed by the equation

$$
\mathbf{H}_{t}[X]=\mathbf{H}_{t}[\mathbf{G}[X, t]]
$$

that together with the initial condition $\mathbf{H}_{0}[X]$ completely determine this distribution.

\section{Kolmogorov's master equation}

On the basis of (3.7), we now obtain the evolution equation of mathematical expectations for the random process $\mathfrak{N}_{\Delta}$. For this we introduce the matrix $s_{l j}(t)=\mathbf{E} \tilde{\mathcal{v}}_{l j}(t)$ of mathematical expectations whose matrix elements are distinguished from zero only at $j \leq l$. It is defined by the formula

$$
s_{l j}(t)=\sum_{k_{j}=0}^{\infty} k_{j} q_{l}\left(k_{j^{\prime}}, j^{\prime} \in \mathbb{N}_{+} ; t\right)=\left(\frac{\partial g_{l}[X, t]}{\partial x_{j}}\right)_{X \equiv 1} .
$$

Further, the mathematical expectation $n_{l}(t)=\mathbf{E} \tilde{\nu}_{l}(t)$ of the number $\tilde{\nu}_{l}(t)$ of fragments with the size $l$ at the time instant $t$ is defined by the generating function $\mathbf{H}_{t}[X]$ by means of its partial derivative in $x_{l}$ at the point $X \equiv 1$,

$$
n_{l}(t)=\mathbf{E} \tilde{\nu}_{l}(t)=\left(\frac{\partial \mathbf{H}_{t}[X]}{\partial x_{l}}\right)_{X \equiv 1}
$$

Then, on the basis of (3.7) and (4.1), we find

$$
n_{l}(t+1)=\left(\frac{\partial \mathbf{H}_{t+1}[X]}{\partial x_{l}}\right)_{X \equiv 1}=\sum_{m=l}^{\infty}\left(\frac{\partial \mathbf{H}_{t}[\mathbf{G}[X, t]]}{\partial g_{m}[X, t]}\right)_{X \equiv 1}\left(\frac{\partial g_{m}[X, t]}{\partial x_{l}}\right)_{X \equiv 1}
$$

that is,

$$
n_{l}(t+1)=\sum_{m=l}^{\infty} n_{m}(t) s_{m l}(t)
$$

Now introduce the functions

$$
N_{l}(t)=\sum_{k=0}^{l} n_{k}(t), \quad S_{m l}(t)=\sum_{k=0}^{l} s_{m k}(t)
$$


8 The Kolmogorov equation in the stochastic fragmentation

Then, by summing up (4.4) for all $l$, we derive the motion equation in terms of this function

$$
\begin{aligned}
N_{l}(t+1)= & \sum_{k=0}^{l} \sum_{m=k}^{\infty} n_{m}(t) s_{m k}(t) \\
= & \sum_{k=0}^{l-1} \sum_{m=k}^{l-1} n_{m}(t) s_{m k}(t)+\sum_{k=0}^{l} \sum_{m=l}^{\infty} n_{m}(t) s_{m k}(t) \\
= & \sum_{m=0}^{l-1} n_{m}(t) \sum_{k=0}^{m} s_{m k}(t)+\sum_{m=l}^{\infty} n_{m}(t) S_{m l}(t) \\
= & \sum_{m=0}^{l-1} S_{m m}(t)\left[N_{m}(t)-N_{m-1}(t)\right]+\sum_{m=l}^{\infty} S_{m l}(t)\left[N_{m}(t)-N_{m-1}(t)\right]
\end{aligned}
$$

where $N_{-1}(t)=0$.

At last, introduce the function $N_{\Delta}(r ; t): \mathbb{R}_{+} \times \mathbb{N}_{+} \mapsto \mathbb{R}_{+}$,

$$
N_{\Delta}(r ; t)=N_{l}(t), \quad \text { if } r \leq l \Delta<r+\Delta .
$$

It is continuous from the left and it is equal to the average fragment number having sizes not greater than $r$. Besides, introduce the function $S_{\Delta}\left(r, r^{\prime} ; t\right): \mathbb{R}_{+} \times \mathbb{R}_{+} \times \mathbb{N}_{+} \mapsto \mathbb{R}_{+}$,

$$
S_{\Delta}\left(r, r^{\prime} ; t\right)=S_{m l}(t), \quad \text { if } r \leq l \Delta<r+\Delta, r^{\prime} \leq m \Delta<r^{\prime}+\Delta
$$

being continuous from the left in both arguments $r$ and $r^{\prime}$. Then for $(l-1) \Delta<r \leq l \Delta$, it follows from (4.6) that

$$
\begin{aligned}
N_{\Delta}(r ; t+1)= & \sum_{m=0}^{l-1} S_{\Delta}\left(r_{m}, r_{m} ; t\right)\left[N_{\Delta}\left(r_{m}+\Delta ; t\right)-N_{\Delta}\left(r_{m} ; t\right)\right] \\
& +\sum_{m=l}^{\infty} S_{\Delta}\left(r_{m}, r ; t\right)\left[N_{\Delta}\left(r_{m}+\Delta ; t\right)-N_{\Delta}\left(r_{m} ; t\right)\right],
\end{aligned}
$$

where $r_{m}=m \Delta$. The sums in the right-hand side of this equality may be considered as integral sums of the Riman-Stiltyes integral for step functions $N_{\Delta}(r ; t)$ and $S_{\Delta}\left(r^{\prime}, r ; t\right)$, that is,

$$
N_{\Delta}(r ; t+1)=\int_{0}^{r-0} S_{\Delta}\left(r^{\prime}, r^{\prime} ; t\right) d N_{\Delta}\left(r^{\prime} ; t\right)+\int_{r-0}^{\infty} S_{\Delta}\left(r^{\prime}, r ; t\right) d N_{\Delta}\left(r^{\prime} ; t\right) .
$$

Assuming that the function $S_{\Delta}\left(r^{\prime}, r ; t\right)$ tends to a continuous function $S\left(r^{\prime}, r ; t\right)$ as $\Delta \rightarrow 0$ and the function $N_{\Delta}(r ; t)$ tends to a monotone nondecreasing function $N(r, t)$ and since discontinuity points of functions $S_{\Delta}\left(r^{\prime}, r ; t\right)$ and $N_{\Delta}\left(r^{\prime}, t\right)$ in the argument $r^{\prime}$ coincide for every $t$, we may apply the second Helly theorem. It permits to realize the limit transition under the integral sign. In this case, we obtain the equation for evolution of average 
fragment number distribution in the form

$$
N(r ; t+1)=\int_{0}^{r-0} S\left(r^{\prime}, r^{\prime} ; t\right) d N\left(r^{\prime} ; t\right)+\int_{r-0}^{\infty} S\left(r^{\prime}, r ; t\right) d N\left(r^{\prime} ; t\right) .
$$

Thus, the following statement takes place.

TheOREM 4.1. Statistical characteristic $N(r, t)=\mathbf{E N}(r, t)$ of the generalized random process $\mathfrak{N}$ is governed by (4.11).

At last, we show that (1.1) of the Kolmogorov theory is a particular case of (4.11). We suppose that the function $S\left(r^{\prime}, r ; t\right)$ depends only on the ratio $r / r^{\prime}$, that is, $S\left(r^{\prime}, r ; t\right)=$ $S\left(r / r^{\prime} ; t\right)$. In this case $(4.11)$ is represented in the form

$$
N(r ; t+1)=S(1 ; t) N(r-0 ; t)+\int_{r-0}^{\infty} S\left(\frac{r}{r^{\prime}} ; t\right) d N\left(r^{\prime} ; t\right)
$$

Applying the integration by parts with the use of conditions $N(\infty ; t)<\infty, S(0 ; t)=0$, we get

$$
N(r ; t+1)=\int_{r-0}^{\infty} N\left(r^{\prime} ; t\right) d S\left(\frac{r}{r^{\prime}} ; t\right)
$$

Introducing the integration variable $k=r / r^{\prime}$, we obtain

$$
N(r ; t+1)=\int_{0}^{1+0} N\left(\frac{r}{k} ; t\right) d S(k ; t),
$$

Unlike (1.1), the latter takes into account the fact that the function $S(k ; t)$ may have a step at the point $k=1$.

\section{Conclusion}

We have shown how the Kolmogorov equation in statistical fragmentation theory may be justified in the framework of a certain probabilistic scheme. At the same time, even in the framework of the construction presented in the work, some general mathematical questions have been still unsolved. For example, it is necessary to clear up under what conditions the limit distribution of probabilistic distributions $q_{l}\left(k_{j}, j \in \mathbb{N}_{+} ; t\right)$ exists and how it should be understood. The simplest situation when we try to answer this question is when this limit should be understood in weak sense. However, it is desirable that this weak limit nevertheless guarantees the existence of random realizations with probability 1. They should be regarded as some finite point random sets on $\mathbb{R}_{+}$.

It is necessary to find some conditions for distributions $q_{l}\left(k_{j}, j \in \mathbb{N}_{+} ; t\right)$ that guarantee the existence of the limit mathematical expectation $\lim _{\Delta \rightarrow 0} \sum_{j \in \mathbb{N}_{+}: j \Delta<r} \mathbf{E} \tilde{\mathcal{v}}_{l j}(t)$ such that it is a continuous function $S\left(r^{\prime}, r ; t\right)$.

Finally, it is very important to prove the existence of the limit characteristic functional $\Psi[u]$ and, moreover, the existence of random trajectories of the process connected with this functional. 
10 The Kolmogorov equation in the stochastic fragmentation

\section{Acknowledgment}

The authors are grateful to RFBR and Belgorod State University for the financial support of this work.

\section{References}

[1] A. F. Filippov, On the particle size distribution at the subdivision, Teoriya Veroyatnostei i ee Primenenie 6 (1961), no. 3, 299-318 (Russian).

[2] A. N. Kolmogorov, On the logarithmically normal distribution law of particle sizes at the subdivision, Doklady Akademii Nauk SSSR 31 (1941), no. 2, 99-101 (Russian).

[3] B. A. Sevast'yanov, Branching Processes, Nauka, Moscow, 1971.

R. Ye. Brodskii: Single Crystal Institute, National Academy of Sciences of Ukraine, Lenin avenue 65, 61001 Kharkov, Ukraine

E-mail address: brodskii@isc.kharkov.ua

Yu. P. Virchenko: Belgorod State University, Pobedy 85, 308015 Belgorod, Russia

E-mail address: virch@bsu.edu.ru 


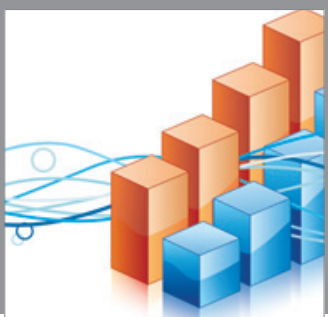

Advances in

Operations Research

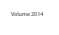

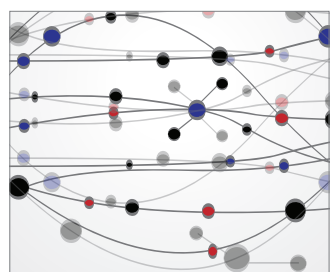

\section{The Scientific} World Journal
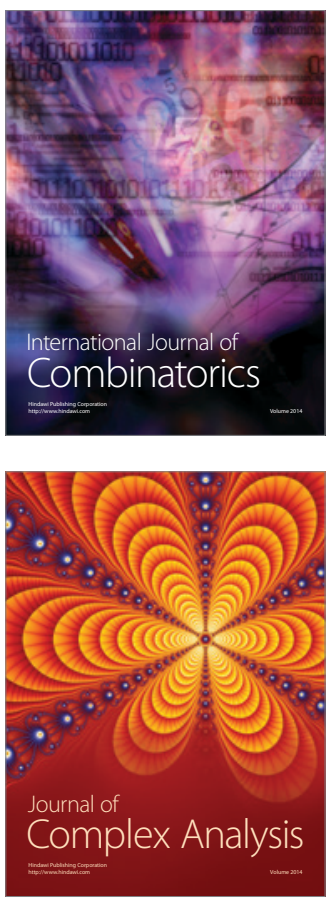

International Journal of

Mathematics and

Mathematical

Sciences
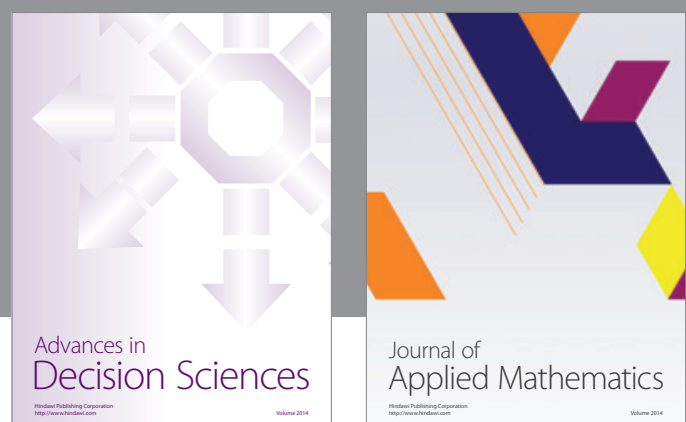

Journal of

Applied Mathematics
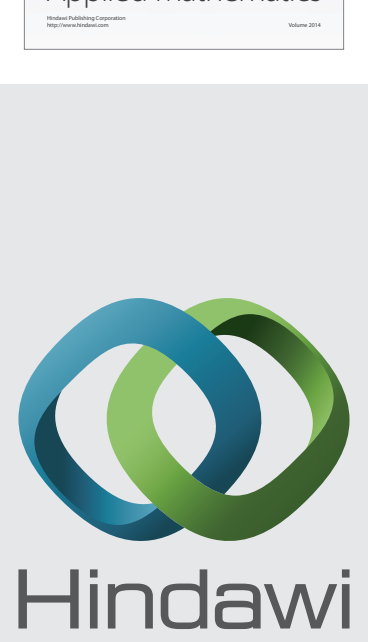

Submit your manuscripts at http://www.hindawi.com
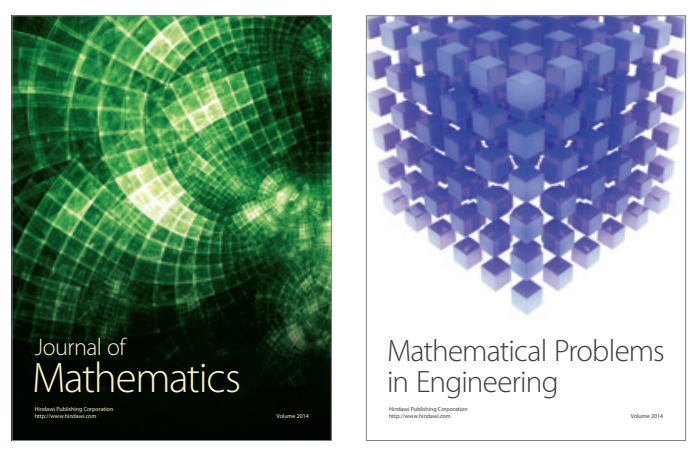

Mathematical Problems in Engineering
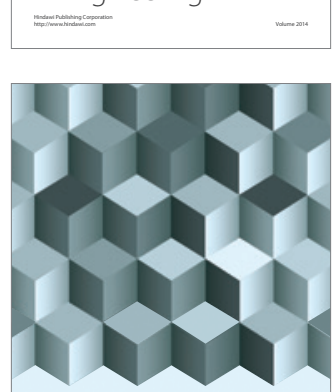

Journal of

Function Spaces
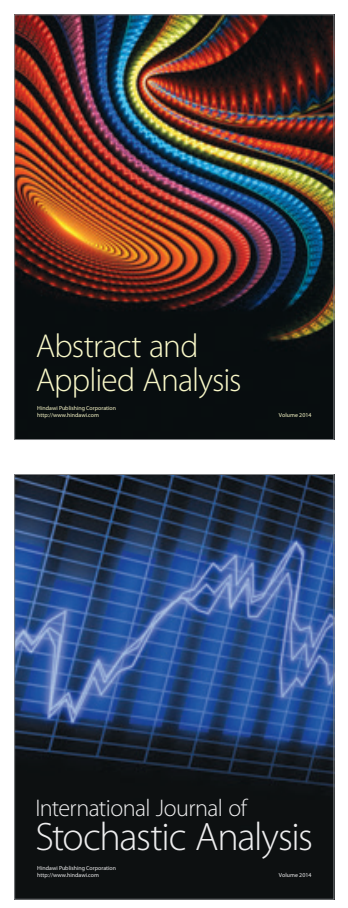

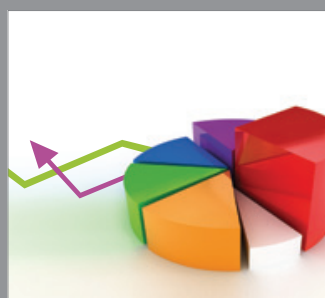

ournal of

Probability and Statistics

Promensencen
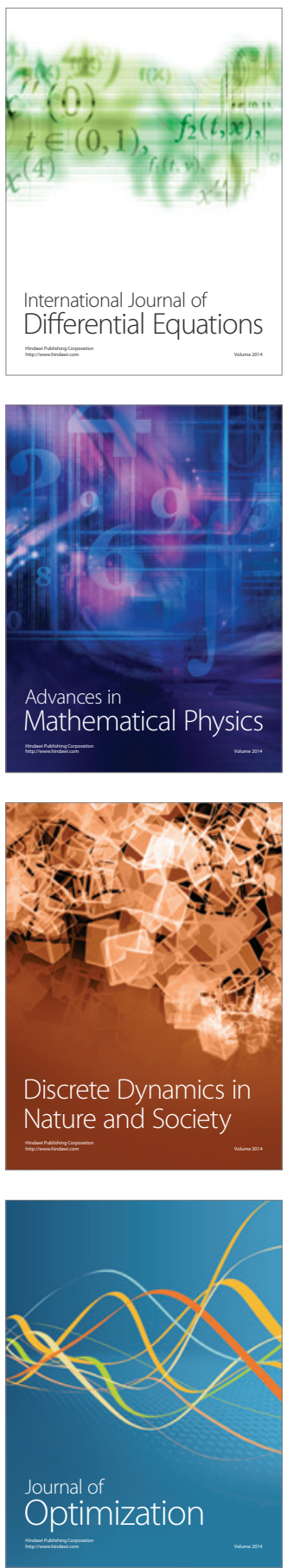